\title{
Protein Folding Simulation with New Move Set in 3D Lattice Model
}

\author{
X.-M. Li \\ Faculty of computer, Guangdong University of technology, Guangzhou, Guangdong, \\ 510006 \\ lxmdwj@163.com
}

\begin{abstract}
We present the lowest energy conformations for several large benchmark problems in 3D HP model. We found these solutions with MC and genetic algorithm using new move set. The new move set including rotation and mirror reflection is suitable for use in protein folding simulation. Experiment results show that new move set can find these best solutions in less time on average and dramatically superior to that of the commonly used move set.
\end{abstract}

Keywords: Protein folding simulation, 3D lattice model, move set.

\section{Introduction}

The HP model for protein folding was introduced by Dill ${ }^{[1]}$. In the model a protein consists of a sequence of amino acids, each labeled as either hydrophobic $(\mathrm{H})$ or hydrophilic (P). The sequence must be placed on a 2D or 3D grid without overlapping so that the adjacent amino acids in the sequence remain horizontally or vertically adjacent in the grid. The goal is to minimize the energy, which in the simplest variation corresponds to maximizing the number of adjacent hydrophobic pairs.

Recent theoretical work has focused on approximation algorithms, although these have not proven helpful for finding minimum energy conformations. Many heuristic algorithms for finding minimum energy conformations have been explored ${ }^{[2] \sim 66}$. In this paper we demonstrate the effectiveness of a new local move set with Monte Carlo and genetic search algorithm on the HP lattice model in protein folding problem.

\section{New Move Set}

A move set in lattice model defines possible conformational changes that can take

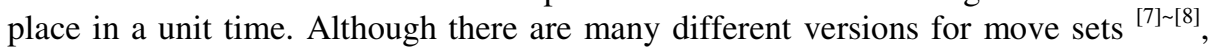
the choice of the move set is not complete solved yet. In the paper, we will extend the previously used move set by adding mirror reflection to the move sets. The new move sets contain four different moves including four-bead crankshaft moves, three bead flip, rotation symmetry and mirror reflection. Moves that violate excluded volume are forbidden. 


\section{Algorithm}

The Monte Carlo method for protein folding can be described in the following general algorithm. (1) Start from a random conformation. (2) From a conformation S1 with energy E1, make a single random change of the conformation to another conformation S2 and evaluate its energy E2. (3) If E2 is less than E1, then accept the change to conformation S2, otherwise decide nondeterministically whether to accept the change according to the energy increase with the change. Usually the criterion is that the new conformation is accepted if: $\operatorname{Rnd}<\exp \left[\frac{E 1-E 2}{c_{k}}\right]$, where Rnd is a random number between 0 and 1 and $c_{k}$ is gradually decreased during the simulation to achieve convergence. If the change was not accepted, then retain the former conformation $\mathrm{S} 1$. (4) If the stop criterion is not met, then repeat steps (2) to (4).

The algorithm (IMC) we applied here is a special implementation of Monte Carlo for the 3D lattice model. We choose new move set as our change in conformation in protein folding.

In improved Genetic algorithms (IGA) of protein folding, each candidate solution is referred to as a set of coordinate value. The process involved in IGA optimization problems is based on natural evolution and works as follows,

(1) Randomly generate an initial population of potential solutions. Each individual must be a legal conformation.

(2) Evaluate the fitness or energy of each solution.

(3) Each conformation of population evolves independently for some iteration by the improved Monte Carlo procedure.

(4) According to crossover probability select two solutions biased in favor of fitness.

(5) Crossover the solutions at a random point on the coordinate string to produce two new solutions.

(6) The lowest energy conformation in current generation is directly replicated to next generation. If the stop criterion is not met, then go back to step (2).

\section{Experience Results}

Table 1 and 2 shows the results of the 10 different 3D 48 mer sequences ${ }^{[9]}$ in $3 \mathrm{D}$ lattice model with Monte Carlo and Genetic algorithm using traditional move set and new move set respectively. For all the sequences, 3 independent simulations are carried out at the same situations. In the table the $\mathrm{N}$ denotes the number of residues of the sequence and $\mathrm{E}$ denotes optimal energies determined from the designed structure. The first item of tables was the obtained minimum energy, the second item was the number of conformations scanned before that energy value was found.

For the ten test cases of 48 mer we choose to start with temperature coefficient $\mathrm{T}=2$ and was cooled by $\mathrm{T}=0.98 \mathrm{~T}$ every 10,000 move steps with Monte Carlo. Genetic algorithm was run for 300 iterations with the population size 200 . For the mutation 
stage the cooling scheme starts with $\mathrm{T}=2$ and cooled by $\mathrm{T}=0.97 \mathrm{~T}$ very 5 generations. The crossover stage starts with $\mathrm{T}=0.3$ and is cooled by $\mathrm{T}=0.99 \mathrm{~T}$ very 5 generations. Bold items in the table show best result per sequence. From the table 1, we know that the Monte Carlo using new move set acquire the lower energy conformations than using classical move set except seq\#1 and seq\#10 which have the same best results. Table 2 indicates that the Genetic Algorithm using new move set find the lower energy conformations than using classical move set except seq\#6 which have the same best results. Because new move set includes not only the classical move set but also mirror reflection, It is not an unexpected result that new move set shows better energy conformation than classical move set. From table 2 we find that IGA methods search the lowest energy conformations for nine of the ten sequences.

Table 1. Results from 3 runs per 3D sequence with MC and IMC

\begin{tabular}{|c|c|c|c|c|c|c|}
\hline \multirow{2}{*}{$\mathrm{N} / \mathrm{E}$} & \multicolumn{3}{|c|}{ MC using classical move set } & \multicolumn{3}{c|}{ IMC using new move set } \\
\cline { 2 - 7 } & 1 & 2 & 3 & 1 & 2 & 3 \\
\hline $1(-32)$ & $-30(798)$ & $\mathbf{- 3 1}(\mathbf{7 4 1})$ & $-29(568)$ & $\mathbf{- 3 1}(\mathbf{7 5 6})$ & $-30(434)$ & $-30(382)$ \\
\hline $2(-34)$ & $\mathbf{- 2 9 ( 6 6 6 )}$ & $-29(737)$ & $-28(614)$ & $-30(650)$ & $\mathbf{- 3 1}(\mathbf{8 9 2})$ & $-30(336)$ \\
\hline $3(-34)$ & $-29(558)$ & $\mathbf{- 3 2}(\mathbf{8 6 2})$ & $-29(434)$ & $-31(629)$ & $\mathbf{- 3 3}(\mathbf{6 2 5})$ & $-32(521)$ \\
\hline $4(-33)$ & $-29(713)$ & $-30(689)$ & $\mathbf{- 3 0}(\mathbf{6 2 0})$ & $\mathbf{- 3 1}(\mathbf{5 4 6})$ & $-31(554)$ & $-30(299)$ \\
\hline $5(-32)$ & $\mathbf{- 2 9}(\mathbf{7 3 1})$ & $-29(770)$ & $-29(793)$ & $-30(761)$ & $\mathbf{- 3 0}(\mathbf{3 0 1})$ & $-30(271)$ \\
\hline $6(-32)$ & $-27(554)$ & $\mathbf{- 2 9}(\mathbf{7 1 6})$ & $-28(617)$ & $\mathbf{- 3 0 ( 1 , 2 4 0 )}$ & $-28(46)$ & $-29(431)$ \\
\hline $7(-32)$ & $-27(546)$ & $\mathbf{- 2 9}(\mathbf{6 1 2})$ & $-28(549)$ & $-29(115)$ & $\mathbf{- 3 0}(\mathbf{6 3 5})$ & $-28(185)$ \\
\hline $8(-31)$ & $\mathbf{- 2 9 ( 7 8 6 )}$ & $-28(771)$ & $-28(932)$ & $\mathbf{- 3 0}(\mathbf{5 0 7})$ & $-28(296)$ & $-29(409)$ \\
\hline $9(-34)$ & $-29(691)$ & $-31(787)$ & $\mathbf{- 3 1 ( 5 7 3 )}$ & $-32(749)$ & $-31(134)$ & $\mathbf{- 3 2}(\mathbf{4 5 1})$ \\
\hline $10(-33)$ & $-30(729)$ & $-31(846)$ & $\mathbf{- 3 2}(\mathbf{1 , 0 2 1})$ & $-30(315)$ & $\mathbf{- 3 2}(\mathbf{4 2 1})$ & $-31(568)$ \\
\hline
\end{tabular}

Table 2. Results from 3 runs per 3D sequence with GA and IGA

\begin{tabular}{|c|c|c|c|c|c|c|}
\hline \multirow{2}{*}{ N/E } & \multicolumn{3}{|c}{ GA using classical move set } & \multicolumn{3}{c|}{ IGA using new move set } \\
\cline { 2 - 7 } & 1 & 2 & 3 & 1 & 2 & 3 \\
\hline $1(-32)$ & $-31(1,503)$ & $-30(1,494)$ & $\mathbf{- 3 1}(\mathbf{1 , 4 5 1})$ & $-31(482)$ & $\mathbf{- 3 2}(\mathbf{6 8 5})$ & $-32(750)$ \\
\hline $2(-34)$ & $\mathbf{- 3 1}(\mathbf{1 , 2 4 2})$ & $-30(1,213)$ & $-30(960)$ & $\mathbf{- 3 4}(\mathbf{8 3 0})$ & $-32(863)$ & $-32(1,192)$ \\
\hline $3(-34)$ & $\mathbf{- 3 2}(\mathbf{9 0 6})$ & $-31(1,304)$ & $-32(1,251)$ & $-33(434)$ & $\mathbf{- 3 4 ( 5 3 5 ) ( 5 6 )}$ & $-34(1,077)$ \\
\hline $4(-33)$ & $-31(1,715)$ & $\mathbf{- 3 1}(\mathbf{1 , 1 8 5})$ & $-30(834)$ & $-32(714)$ & $-31(660)$ & $\mathbf{- 3 3 ( 1 , 2 1 2 )}$ \\
\hline $5(-32)$ & $\mathbf{- 3 1}(\mathbf{1 , 1 9 6})$ & $-30(950)$ & $-31(1,501)$ & $-31(560)$ & $\mathbf{- 3 2 ( 8 3 1 )}$ & $-31(1,101)$ \\
\hline $6(-32)$ & $\mathbf{- 3 1}(\mathbf{2 , 1 7 6})$ & $-30(873)$ & $-30(1,326)$ & $-31(984)$ & $-30(365)$ & $\mathbf{- 3 1}(\mathbf{9 5 8})$ \\
\hline $7(-32)$ & $-30(1,538)$ & $\mathbf{- 3 0}(\mathbf{1 , 2 6 0})$ & $-29(1,474)$ & $\mathbf{- 3 2}(\mathbf{1 , 0 9 1})$ & $-30(458)$ & $-31(715)$ \\
\hline $8(-31)$ & $-29(1,205)$ & $-29(1,314)$ & $\mathbf{- 3 0}(\mathbf{1 , 3 0 0})$ & $-30(1,081)$ & $-31(913)$ & $\mathbf{- 3 1}(\mathbf{7 5 6})$ \\
\hline $9(-34)$ & $-31(1392)$ & $\mathbf{- 3 2}(\mathbf{2 , 3 1 2})$ & $-31(1,256)$ & $-31(482)$ & $\mathbf{- 3 2}(\mathbf{6 8 5})$ & $-32(750)$ \\
\hline $10(-33)$ & $-31(1,737)$ & $\mathbf{- 3 2}(\mathbf{1 , 3 4 5})$ & $-31(953)(99)$ & $\mathbf{- 3 4}(\mathbf{8 3 0})$ & $-32(863)$ & $-32(1,192)$ \\
\hline
\end{tabular}

The results of IGA were summarized and compared with other methods in Table 3. The data item of IGA and IMC of Table 3 come from the best results of table 2 and table 1 respectively. The data item of MC comes from reference [9].

From the table 3 we find that new move set is superior in protein folding simulations. IMC method finds lower energy conformation than $\mathrm{MC}$ in six of ten sequences and two sequences obtain the same local minima and only two sequences inferior to MC method. 
Table 3. Comparison IMC and IGA with other methods in 3D lattice model

\begin{tabular}{|c|c|c|c|c|c|c|c|}
\hline N/E & IGA & IMC & MC & N/E & IGA & IMC & MC \\
\hline $48-1(-32)$ & $-32(685)$ & $-31(756)$ & -30 & $48-6(-32)$ & $-31(958)$ & $-30(1,240)$ & -30 \\
\hline $48-2(-34)$ & $-34(830)$ & $-31(892)$ & -30 & $48-7(-32)$ & $-32(1,091)$ & $-30(635)$ & -31 \\
\hline $48-3(-34)$ & $-34(535)$ & $-33(625)$ & -31 & $48-8(-31)$ & $-31(756)$ & $-30(507)$ & -31 \\
\hline $48-4(-33)$ & $-33(1,212)$ & $-31(546)$ & -30 & $48-9(-34)$ & $-34(711)$ & $-32(451)$ & -30 \\
\hline $48-5(-32)$ & $-32(831)$ & $-30(271)$ & -30 & $48-10(-33)$ & $-33(991)$ & $-32(421)$ & -30 \\
\hline
\end{tabular}

\section{Conclusion}

We proposed a new move set and carried out comparative studies on the effects of move sets in protein folding in the cubic lattice model. It would like to expand our prototype to handle more challenging protein folding problems. We can conclude that GA and MC simulation of lattice protein folding is highly dependent on the move set used. In the folding simulation a more flexible move set always results in faster folding and lower energy conformation than commonly used move set. With appropriate modifications and enhancements, we expect the method to be useful for dealing with folding simulation of real protein sequence.

\section{References}

1. Ken A..Dill. Theory for the folding and stability of globular proteins. Biochemistry, (1985)24:1501

2. S. Kirkpatrick, C. D. Gelatt, Jr., and M. P. Vecchi, Optimization by simulated annealing. Science (1983)220:671

3. Ron Unger and John Moult. Genetic algorithms for protein folding simulations. J.Mol. Biol. (1993)231:75

4. Rainer Konig and Thomas Dandekar. Improving genetic algorithms for protein folding simulations by systematic crossover. Biosystems. (1999) 50: 17 25

5. Faming Liang. Evolutionary Monte Carlo for protein folding simulations. J.Chem.Phys. (2001) 115:3374

6. Tianzi. Jiang Protein folding simulations of the hydrophobic-hydrophilic model by combining tabu search with genetic algorithms. J.Chem.Phys. (2003)119:4592

7. J.Shin, W.S. Oh. Study of move set in cubic lattice model for protein folding. J.Phys.Chem. (1998) 102:6405 6412.

8. N.L. Nunes, K. Chen, J.S. Hutchinson, A flexible lattice model to study protein folding, J.Phys. Chem. (1996) 100:10443.

9. Yue K and Fiebig KM. A test of lattice protein folding algorithms. Proc Natl Acad Sci USA. (1995) 92:325 